\title{
Marco curricular y legislativo de un proyecto de centro de educación para el desarrollo bajo principios de transversalidad y sentido de comunidad
}

\section{Curricular and legislative framework of a school project of development education with transversality and community principles}

\author{
M del Camino Pereiro González*, Ma Beatriz Páramo Iglesias* \\ * CPI Plurilingüe Virxe da Cela
}

\begin{abstract}
Resumen
En esta comunicación, presentamos el diseño y las características pedagógicas y didácticas del proyecto titulado "A vaquiña polo que vale", y su encuadre en el contexto del colegio público integrado (CPI) plurilingüe Virxe da Cela, en los diferentes niveles de concreción curricular, planes y proyectos de centro, así como también en el marco curricular de las diferentes etapas educativas en las que se insiere este proyecto en el centro. Su finalidad última es la educación para el desarrollo desde el quehacer y contexto local para abordar temáticas globales y de interés para la comunidad educativa. Para ello se presenta la descripción y puntos fuertes del proyecto para visualizar una secuencia educativa que intenta aunar comunidad educativa y educación para el desarrollo con carácter transversal.

Palabras clave: educación para el desarrollo, comunidad, proyecto, innovación, aprendizaje a lo largo de la vida.
\end{abstract}

\begin{abstract}
In this communication, we present the design and pedagogical and didactic characteristics of the project entitled "A vaquiña polo que vale", and its setting in the context of the Colegio Público Integrado (CPI) Plurilingüe Virxe da Cela, in the different levels of curricular concretion, Plans and projects of the center, as well as in the curricular framework of the different educational stages in which this project is inserted. Its purpose is the education for development from the local context and to tackle global issues of interest to the educational community. For this, the description and strengths of the project are presented to visualize an educational sequence that tries to combine educational community and education for development with transversal character. Keywords: Education for development, community, project, innovation, lifelong learning.
\end{abstract}

\section{Punto de partida}

El CPI Plurilingüe Virxe da Cela está inmerso en un proceso de cambio y mejora con diferentes planes y proyectos cuyo objetivo es dar respuesta a la necesidad imperante de un desarrollo endógeno sostenible que establezca actuaciones que permitan identificar las fortalezas del medio y del contexto de toda la comunidad educativa del centro. En todas las actuaciones del centro y en el proyecto que se presenta prima en este caso el valor humano de la educación, y de la importancia de trasladar la vida cotidiana a la escuela. El objetivo del proyecto que se está llevando a cabo desde el curso académico 2015/2016 es dotar de importancia las transiciones que se dan a diario entre escuela y contexto, la vida del alumnado, de las familias, de los docentes, de los demás agentes y personas de la escuela, y aún yendo más allá, de los vecinos, del contexto, de la asociación y de la universidad como punto de partida para un aprendizaje real y contextualizado, que atienda a la diversidad, necesidades y fortalezas de cada uno y de cada contorno. El eje central de dicho proyecto para facilitar los aprendizajes "que van y vienen" son el sector lácteo y ganadero de la zona.

Las líneas de investigación actuales (Gutiérrez, Benayas y Calvo, 2006) abogan por estudios que no sólo atiendan a la educación para el desarrollo y sus vertientes y ejes desde lo global, sino también y primeramente desde lo local, partiendo de lo cercano para poder entender grandes problemáticas del mundo desde nuestros ojos y nuestro contorno que mucho tienen que ver con esos dos ejes del proyecto (sector lácteo y ganadero). Así, uno de los dilemas que se planteó al idear, proyectar y programar un proyecto de esta envergadura desde el CPI Virxe da Cela y su comunidad educativa, la Asociación Solidariedade Internacional de Galicia y la Universidad de la Coruña fue encuadrar todos sus ejes, prácticas, y vertientes en el marco legislativo actual y que está inmerso en un momento de permuta en muchas de sus etapas, siguiendo experiencias llevadas a cabo con esta filosofía (Aguilera, Mendoza, Racionero y Soler, 2010).

\section{Descripción del proyecto}

A continuación se expone un breve resumen del proyecto, objetivos a los que intenta responder y la implicación de los diferentes agentes de la comunidad educativa, punto fuerte de dicho proyecto. 


\section{Resumen del proyecto}

Se trata de dotar al alumnado y a los demás agentes educativos de las destrezas, habilidades, capacidades personales y sociales necesarias para reinventar el rural gallego desde el respeto, conocimiento, valoración del medio en el que vivimos como punto de partida para aprender en cualquier contexto a lo largo de nuestras vidas y que permitan transiciones y aprendizajes adaptadas y contextualizas con sentido también de lo global. Es decir el desarrollo de valores y actitudes de ciudadanía global crítica y responsable (Ed. para el desarrollo y la ciudadanía global).

\section{Objetivos generales y específicos}

El objetivo principal ha sido motivar a la Comunidad Educativa en el diseño y desarrollo de un proyecto común de trabajo que se asiente en la Educación para el Desarrollo y la Ciudadanía Global. Y los objetivos específicos de este proyecto fueron:

- Conocer y valorar el contorno y vida cotidiana de nuestro centro como punto de partida para una educación personalizada y para toda la vida

- Acercarse a diferentes problemáticas del entorno con una visión local y global de los mismos desde una visión crítica y constructiva

- Proponer actuaciones y actividades que permitan desarrollar habilidades de pensamiento y aprendizaje en la que participe toda la comunidad educativa y organismos vinculados.

\section{Población participante y destinatarios del proyecto}

El desarrollo del proyecto supuso un cambio en las dinámicas del centro, tanto a nivel organizativo como pedagógico y de relación con la sociedad en general, se convirtió, junto con el proyecto "Monfero Solidario" y "Somos paisaje y vida" en un referente de los valores del mismo. A nivel organizativo, estableciendo vías efectivas de coordinación docente; a nivel pedagógico, la asunción de propuestas pedagógicas innovadoras (trabajo por proyectos, tareas de aprendizaje o aprendizaje servicio) asentadas en la investigaciónacción y en el trabajo cooperativo incluso entre etapas; y a nivel social, al establecerse como elemento dinamizador y de cohesión educativas y social y dar a conocer el contorno y su valor. En definitiva, contexto y comunidad educativa refuerzan lazos para un desarrollo integral del alumnado y para la puesta en común de valores de solidaridad, responsabilidad y acción social.

Descripción y grado de implicación de los y las docentes. Desde el inicio del proyecto se unen al mismo 26 docentes (98\%), prácticamente la totalidad del profesorado. La organización de las actividades y su desarrollo se realiza a partir de la reflexión crítica del contexto y sus problemáticas. En primer lugar se establece la integración curricular de las acciones un proyecto común (ABP que se centra principalmente en el aprendizaje de servicio solidario -ApsS). Empezamos el diseño mediante el establecimiento de una propuesta de acción común a partir del contexto y de los intereses y motivaciones de los estudiantes. ¿En qué realidad nos enmarcamos? ¿Cuáles son las demandas de nuestro alumnado? ¿Qué potencialidades tenemos? ¿Cuáles son nuestras problemáticas? Estas, ¿son parecidas a las de otros contextos? ¿Qué futuro queremos? fueron interrogantes establecidos como punto de partida. A partir de las mismas, especificamos como se implementarían desde un punto de vista pedagógico, organizativo y de gestión, incluyendo tanto la secuenciación temporal y como su desarrollo curricular en las diferentes etapas educativas. Estamos hablando de una metodología de proyectos integrados con un producto final relevante que sean quien de aprovechar los recursos del centro desde la funcionalidad comunitaria y solidaria (aprendizaje servicio solidario) y además configurar una verdadera Educación para o Desarrollo y la Ciudadanía Global (EpDCG). Para un adecuado desarrollo del proyecto, contamos con la formación específica del profesorado, tanto individual, como en diferentes grupos de trabajo y con PFAC de línea de convivencia y mejora da calidad educativa del centro, así como a que parte de otras instituciones relevantes.

Descripción y grado de implicación de la comunidad educativa. Creemos en el esfuerzo compartido, por lo que tanto personal docente como no docente se configuraron como incentivadores y colaboradores de las diferentes actuaciones. Personal de cocina, transporte escolar, conserje y administrativa ejercieron de informantes y colaboradores en la organización de actividades. Las familias son una parte fundamental en el proyecto.

Para lograr su implicación se potencia la comunicación y la vías de participación (dinámicas de centro, reuniones regulares AMPA, creación la Escuela de Familias, Espacio Familias (0-3), apertura de la biblioteca del centro, salidas a granjas y queserías de las familias de la comarca, Radio- intergeneracional, etc). Así conocieron y participaron en la integración del proyecto a nivel curricular y en su desarrollo metodológico y evaluativo de las actuaciones. Se trataba de establecer un proyecto comunitario con valor y transcendencia social.

A partir de las actuaciones, consideramos que posibilitamos cambiar las dinámicas de centro, tanto a nivel organizativo como pedagógico y en relación con la sociedad en general, convirtiéndose las diferentes iniciativas, junto con el proyecto "Monfero Solidario y "Somos paisaxe, somos vida" en un referente de los valores del mismo, revertiendo tanto en el futuro del alumnado, como en el del centro y de la comunidad educativa. Ejemplo de esto es que a partir de las diferentes actuaciones, se consolido nuestra colaboración con diferentes $\mathrm{ONG}^{\prime}$ s. Este proyecto nace de la colaboración con la Universidad de la Coruña y con las ONG's Solidariedade Internacional $\mathrm{Y}$ Cooperación Galega con el objetivo abordar la introducción del paradigma de la Dimensión Global da Educación para el Desarrollo en el contexto escolar de una forma transversal. También fundamental fue contar con la colaboración de diferentes instituciones públicas y privadas (Consellaría de Medio Rural e do Mar, Dirección Xeral de Xuventude, Ayuntamientos de Monfero e Irixoa, , USC, ONGs, Confederación Anpas Galegas, Consellaría de Educación, entre otras) que fornecen y dotan de significado para el alumnado y la 
comunidad en general al proyecto. Participamos en el programa de Acción Solidaria en España de la Fundación Ayuda en Acción, contando gracias al mismo con financiación para realizar salidas didácticas complementarias que permiten al alumnado trasladar las habilidades, destrezas y competencias trabajadas a contextos reales. Igualmente participamos de la actuación de innovación educativa Genios en colaboración con Google (programación computacional) y de desarrollo de la competencia matemática (Smartick) y de la competencia lingüística (Glifing). Además de esto, pertenecemos a la Rede Solidaria da Mocidade de Entreculturas, participando activamente, tanto alumnado como profesorado en las actividades formativas y divulgativas como en el voluntariado. De forma permanente, son 35 alumnos y alumnas los que participan en las acciones de voluntariado. Igualmente, somos partícipes del proyecto "School to School" de la Fundación Vicente Ferrer de intercambio cultural entre escuelas españolas y otras del mismo nivel en la India. A través de este intercambio, el programa busca que los discentes tomen conciencia de las desigualdades y aprendan valores de solidaridad e de justicia social. Siguiendo con estas dinámicas, destacar las colaboraciones con Cruz Vermella, con la Cocina Económica de Ferrol, con la Fundación Seur, con Recoatlántico, con Glorre Global Humanitaria, entre otras. También cabe citar la participación y colaboración con otros centros próximos.

Descripción y grado de implicación del alumnado. Los discentes son los encargados de concretar las actuaciones partiendo de que debe ser quien de suscitar una reflexión crítica sobre las actuaciones del ser humano en el medio en el medio en el que se desarrolla. Ante esto, se estableció un reto significativo que, tras una posta en común, se concreto en objetivos viables. ¿Cómo es nuestra comarca? ¿Cómo nos desarrollamos como sociedad? ¿Qué relación mantenemos con el medio natural? ¿Qué futuro queremos? Así nace el análisis y la puesta en valor del sector lácteo en la comarca, no solo desde el punto de vista económico, sino también en relación con el medio natural, la cultura, lo social, la coeducación, las relaciones intergeneracionales, la diversidad y el enfoque global a través de su traslado a otras realidades. Es el proyecto "A vaquiña polo que vale"

\section{Planificación del proyecto}

A partir de aquí, comienza el trabajo cooperativo y solidario del alumnado, siendo los docentes (uno o dos coordinadores por actividad) mediadores, facilitadores de recursos y catalizadores de las disertaciones. No menos importante fue conseguir la participación y colaboración del resto da comunidad educativa, participando de las actuaciones e implicándose en su desarrollo. Por lo tanto, estamos ante una propuesta metodológica colaborativa y participativa (alumnado protagonista que selecciona las problemáticas a trabajar), asentada en un diseño abierto, flexible y susceptible de las precisas modificaciones (discentes que cooperan, crean y reinventan en función de las necesidades y problemáticas), capaz de conseguir el interés y la motivación do alumnado (son artífices activos que involucran al resto de la Comunidad), con un tratamiento globalizado e interdisciplinar de las diferentes áreas y materias educativas y de la educación en valores, adaptada a la diversidad del alumnado y facilitadora de la implicación de la comunidad educativa (productos finales relevantes a nivel social). Se crea la figura del Voluntariado de centro y de la tutoría entre iguales. Hablamos de la creación de proyectos, tareas y experiencias educativas integradas, donde destacamos propuestas innovadoras en las que prima sobre todo, el aprendizaje -servicio solidario enfocada cara la valorización del medio natural y de las relaciones que en él se establecen como fórmula de desarrollo endógeno. Se trata de fortalecer la identidad cultural asentada en el medio natural desde una perspectiva global de desarrollo (pensar globalmente para actuar localmente) para ser quien de crear procesos endógenos de respecto, conservación e valorización del paisaje, entendido como desde su dimensión natural, social y cultural. Además, los productos finales relevantes deben ser quien de suponer transcendencia, es decir, repercusión en el medio y también en el contexto. Aquí, es fundamental el tratamiento de las tecnologías da información e a comunicación (TIC) tradicionales (talleres de radio) o emergentes (programación, edición de imagen, vídeos, entre otras) para crear un verdadero espacio tecnológico de aprendizaje e conocimiento (TAC).

\section{Cronograma}

El proyecto se conforma de 3 grandes fases en la que el trabajo se realizó de manera conjunto y coordinada con los agentes participantes de la escuela, universidad y ONG. La primera de ellas realizada durante el curso académico 2015-2016 y que consistió en:

- Revisión y búsqueda bibliográfica. Delimitación del objeto de estudio, problemática y preguntas de investigación.

- Preparación del escenario. Detección de necesidades. Definición de participantes. Análisis de entorno y documento de centro

- Planificación y estructuración del proyecto en tres grandes bloques y centros de interés.

La segunda se centró en el desarrollo y puesta en marcha de las actividades propuestas durante el curso académico 2016-2017 con las siguientes líneas de actuación.

- Delimitación de actividades y cronograma

-Elaboración de instrumentos de investigación, escalas de observación y rúbricas, y selección de técnicas de investigación: análisis de contenido, grabaciones, entrevistas...

- Puesta en marcha de las actividades y recogida de datos.

Y la tercera fase se realizará en el curso 2017-2018 cuyo fin será:

- Análisis de datos recogidos: análisis descriptivo, comparativo y de contenido e interpretación de datos, propuesta de mejora, conclusiones, limitaciones y proyección del estudio.

- Difusión del proyecto y publicación de los datos. 


\section{Actividades}

Dos fueron las actividades iniciales para el lanzamiento de la parte práctica del proyecto. Una fue la actividad que se llevó a cabo en Educación Infantil que consistía en hacer tantas preguntas como les fuera posibles al alumnado de esta etapa sobre la leche y la vaca, eje central del proyecto. La idea que se lanzó al centro fue de si el resto de alumnado y comunidad educativa sería capaz de contestar a todas esas preguntas. Y la otra se llevó a cabo unas jornadas en el centro de Educación para el desarrollo para iniciar en el tema a toda la comunidad implicada.

A continuación se citan algunas de las actividades realizadas y a la pregunta que intentaban contestar así como los diferentes bloques de trabajo a los que pertenecía y etapa en la que se realizo (ESO: Educación Secundaria Obligatoria, EP: educación primaria y EI: educación infantil).

\section{La leche en la sociedad}

\begin{tabular}{ll}
\hline & \multicolumn{1}{c}{ ¿Hay que tirar la leche? } \\
\hline ESO & $\begin{array}{l}\text { Recopilación y análisis de anuncios del sector } \\
\text { lácteo }\end{array}$ \\
\hline EI & Análisis de publicidad \\
\hline EP & $\begin{array}{l}\text { Entrevista a las familias } \\
\text { https://youtu.be/ixajbm3nzhk }\end{array}$ \\
\hline Todo o & $\begin{array}{l}\text { El proyecto de la leche llena nuestro carnaval de } \\
\text { color } \\
\text { https://youtu.be/an7n6gvyg9s } \\
\text { https://youtu.be/gy73kwwzqma }\end{array}$ \\
\hline
\end{tabular}

\section{La vida en la explotación}

¿Cómo se llaman las vacas? ¿Por qué tienen esos nombres? ¿Cómo se les “afala”?

\begin{tabular}{|c|c|}
\hline EI & Diccionario de los nombres de nuestras vacas \\
\hline \multicolumn{2}{|r|}{ ¿A dónde va la leche cuando sale de la vaca? } \\
\hline ESO & La evolución tecnológica del sector \\
\hline EP & $\begin{array}{l}\text { La evolución tecnológica del sector a través de las } \\
\text { imágenes } \\
\text { Excursión a una explotación ganadera y a una } \\
\text { quesería } \\
\text { Programa de radio sobre la evolución del sector } \\
\text { ganadero } \\
\text { Programación y robótica }\end{array}$ \\
\hline EI & $\begin{array}{l}\text { Dibujamos el proceso de la leche } \\
\text { Excursión a una quesería }\end{array}$ \\
\hline \multicolumn{2}{|r|}{ ¿Qué come? } \\
\hline EP & $\begin{array}{l}\text { Impacto } \\
\text { (https://youtu.be/0rWKwxo-r4U }\end{array}$ \\
\hline EI & Creación de vitaminas para las vacas \\
\hline \multicolumn{2}{|r|}{ ¿Hay vacas malas? } \\
\hline EI & Decálogo del uso de las vacas \\
\hline \multicolumn{2}{|r|}{ ¿Todas las vacas son blancas? } \\
\hline $\begin{array}{l}\text { Todo el } \\
\text { Centro }\end{array}$ & $\begin{array}{l}\text { Desfile de } \\
\text { https://youtu.be/ewhBeRcPAis) } \\
\text { Creación del logotipo del proyecto } \\
\text { (https://youtu.be/1EZvy3Vz-yc) }\end{array}$ \\
\hline
\end{tabular}

La leche en la dieta

\begin{tabular}{ll}
\hline & ¿Qué ponen las pegatinas de los cartones de leche? \\
\hline EI & Creación de la etiqueta perfecta \\
\hline \multicolumn{2}{c}{ ¿Hay que beber toda la leche? } \\
\hline ESO & $\begin{array}{l}\text { Análisis de valores nutricionales de productos } \\
\text { lácteos }\end{array}$ \\
\hline EI & Análisis de ingredientes de productos lácteos \\
\hline EP & Libro de recetas con leche \\
\hline $\begin{array}{l}\text { Todo el } \\
\text { Centro }\end{array}$ & La leche en las culturas del centro \\
\hline
\end{tabular}

\section{Evaluación del proyecto}

Estamos ante un elemento fundamental e inseparable de la práctica educativa que, de forma global y sistemática, nos permite recoger, en cada momento, la información necesaria para poder realizar los juicios de valor oportunos que facilitaran la toma de decisiones respecto del desarrollo del proceso. No implica solamente al alumnado, sino también a la pluralidad de agentes que intervienen en esta acción educativa. Valoramos el progreso del alumnado en relación con el punto de partida, teniendo en cuenta las singularidades individuales para observar los conocimientos, habilidades, destrezas, actitudes, hábitos de trabajo e implicación en las dinámicas establecidas, así como a su repercusión en el contexto. Se diseñaron instrumentos de evaluación ajustados a las características del contexto y coherentes con la metodología empleada (observación del trabajo diario, análisis de las actuaciones, informes, rúbricas, escalas, , entrevistas y anotaciones sistemáticas o esporádicas, evaluación figuroanalógica).

En cuanto a la labor docente, alcanza a la recogida de información sobre la consecución de objetivos, los recursos empleados, la temporalización, los espacios, la metodología, al adecuación de la programación a las necesidades del alumnado, aspectos que hagan referencia a las relaciones con el equipo docente, con la familia... No menos importante es evaluar la adecuación a los diferentes documentos del centro (PE, PXA, Propuesta pedagógica, Proyecto lector, Proyecto lingüístico, Plan de convivencia, plan TIC, Plan de acción litoral, entre otros), y su relación con el proyecto como señalaba ya Antúnez (1998).

En cuanto a los indicadores de logros establecidos para la evaluación del proyecto y para la toma de decisiones respecto a los resultados obtenidos, a partir de los mismos, se diseñan instrumentos específicos de evaluación que permita graduar el nivel de consecución de dichos objetivos (escalas de observación, encuestas y firmas).

Respeto al proyecto y a la actuación docente, el proyecto se adecúa a los principios establecidos en el Proyecto Educativo del centro. Y su diseño es coherente con los objetivos diseñados y adecuándose a los diferentes planes y proyectos del centro (partimos de la necesidad y de la realidad de configurar los recursos del medio como elemento de primer orden.)

La selección y secuenciación de los elementos curriculares de la programación del aula respeto al proyecto, cuentan con una distribución y progresión adecuada a las características del alumnado (las actuaciones diseñadas están acordes con los elementos 
curriculares y son quienes de facilitar el desarrollo competencial del alumnado y profesorado -concreción curricular del centro.)

La organización de las actividades es flexible, con actividades y recursos (personales, materiales, temporales, espaciales y agrupamientos adecuados (la vida del centro respira alrededor de las actuaciones, adecuándonos tanto al nivel curricular como organizativo -biblioteca, actividades complementarias, teniendo repercusión a nivel social, tendiendo especial relevancia los órganos de coordinación docente y dinamizadores).

La intervención educativa es apropiada y acorde con la metodología diseñada (reconocimiento institucional) Las actividades aseguran la adquisición de los objetivos didácticos previstos, el desarrollo competencial (trabajo cooperativo, resolución de problemas, iniciativa personal, entre otros) y una adecuada atención a la diversidad ( $100 \%$ de titulación del alumnado del centro.) El profesorado participa en las actividades formativas y de difusión activamente y traslada los aprendizajes a la práctica docente (Plan de formación en centros y formación en otros ámbitos educativos- universidades, movimientos de renovación pedagógica, asociaciones, ONGs, asociaciones de Anpas Gallegas).

Respeto del alumnado, es quien de establecer una secuencia adecuada de la propuesta problemática sugerida, teniendo en cuenta la formulación del problema, los resultados esperados, los datos disponibles, las restricciones y los procesos necesarios (desarrollo de técnicas de trabajo cooperativo y resolución de problemas adquiridos a partir del trabajo por los proyectos, entendiendo los retos como un esfuerzo compartido).

El producto final se ajusta a los objetivos marcados y cuenta con repercusión efectiva según los parámetros de calidad establecidos (el producto final es relevante y el alumnado valora los resultados y participa en la difusión de las actuacionesJornadas de Voluntariado, seminarios, informes $y$ talleres en la USC y en la UDC, Ayuntamientos, entre otros). El alumnado evidencia la adquisición de las competencias necesarias para continuar su progreso académico y personal aplicando los valores adquiridos (en el centro, el alumnado crea la Asociación "Soño Rural" que se encarga de dar valor y difundir las actuaciones- incluídas dos mociones de apoyo al medio rural en los Ayuntamientos de Irixoa y Monfero, así como o "Voluntariado de Centro").

Las dinámicas de trabajo cooperativo están bien organizadas, integrándose las aportaciones individuales de forma reflexiva y consensuada, con una clara definición de tareas y asunción de roles, cumpliéndose los términos de presentación de los trabajos. El alumnado participa activamente en la actuaciones, con interés, motivación, empatía, responsabilidad y espíritu cooperativo y emprendedor.

$\mathrm{Y}$ respeto a las familias y la comunidad educativa en general, las vías de comunicación y participación de las familias fueron adecuadas (participación activa en las iniciativas y colaboración del Anpa en el desarrollo de las mismas). Las familias conocen la integración del proyecto a nivel curricular y el desarrollo metodológico y evaluativo de las actuaciones (reuniones informativa, circulares, colaboración en los procesos). Los canales de cooperación con otras instituciones resultaron efectivas (colaboración con la Consellería de Medio Rural

Dirección Xeral de Xuventude e Voluntariado, universidades, asociaciones, ONGs, entre otras).

\section{Sostenibilidad del proyecto}

Contamos con iniciativas que sustentan este proyecto "Monfero Solidario" y "Somos paisaxe, somos vida" asentando la educación para el desarrollo y ciudadanía global. Este planteamiento no se configuro como nuestra primeira apuesta, sino como acciónes puntuales llevadas a cabo (como el Plan Proxecta, colaboraciones con diferentes institución) que sirvieron del sustento pedagógico necesario para convertirse en proyectos de centro consensuados y dialogados por toda la Comunidad. Así se crea este proyecto educativo firme y sólido que atiende al contexto y alumnado con perspectiva de continuidad en el tiempo durante dos cursos académicos inicialmente

\section{Consideraciones finales}

El fin último que se extrapola de este análisis exhaustivo de la justificación y necesidad de proyectos como este con sentido de desarrollo, transición, aprendizaje a lo largo de la vida es, principalmente dotar al alumnado y a los demás agentes educativos de las destrezas, habilidades, capacidades personales y sociales necesarias para reinventar el rural gallego desde el respeto, conocimiento, valoración del medio en el que vivimos como punto de partida para aprender en cualquier contexto a lo largo de nuestras vidas y que permitan transiciones $\mathrm{y}$ aprendizajes adaptadas $\mathrm{y}$ contextualizas con sentido también de lo global. Una vez presentado el marco que encuadra

\section{Referencias}

Aguilera, A., Mendoza, M., Racionero, S., \& Soler, M. (2010). El papel de la universidad en Comunidades de Aprendizaje. Revista Interuniversitaria de Formación del Profesorado, 24

http://www.redalyc.org/articulo.oa?id=27419180004

Antúnez, S. (1998). Claves para la organización de centros escolares. Barcelona: Editorial Hosori.

Gutiérrez, J., Benayas, J., \& Calvo, S. (2006). Educación para el desarrollo sostenible: evaluación de retos y oportunidades del decencia 2005-2014. Revista Iberoamericana de educación, 40, 25-69. http://www.educandonline.com.ar/biblioteca virtual/ Material\%20bibliografico\%2013\%20general.pdf

\section{Agradecimientos}

A toda la comunidad educativa del CPI Virxe da Cela, a la Universidad de A Coruña y su profesorado implicado en el proyecto y a la ONG Solidaridad Internacional de Galicia.

Este trabajo se encuentra inmerso en el proyecto Investigando la dimensión global de la educación para el desarrollo: un estudio piloto en una escuela gallega, que cuenta con la subvención de la Xunta de Galicia. 